\title{
Platinum Group Elements in Road Environments: A Case Study of Poland
}

\author{
Ewa Adamiec \\ AGH University of Science and Technology \\ Al. Mickiewicza 30, 30-059, Kraków, Poland \\ eadamiec@agh.edu.pl
}

\section{Extended Abstract}

Considering anthropogenic sources, several recent studies suggest that road traffic is regarded as one of the major sources of environmental pollution in urban areas in many European cities. PGEs can be easily released to the environment and pose a risk to the environment and adverse human health effects. In several studies it could be shown that PGE fine particles were co-emitted by exhausted fumes from vehicles and accumulate in different environmental compartments directly close to roads. There are a number of processes associated with traffic such as emission, surface abrasion of the catalyst during car operation, turbulence and resuspension that can affect road dust environments. There is still a number of uncertainties relating to the physical properties and chemical characteristics of particles derived from vehicles. Pt, Pd and $\mathrm{Rh}$ are being extensively used in exhaust catalytic convertors. PGE has been reported to bioaccumulate in the environment and cause allergic reactions and various health problems to humans. Significant correlation of $\mathrm{Pt}, \mathrm{Pd}, \mathrm{Rh}$ and positive correlations with $\mathrm{Ce}, \mathrm{Zr}$, Hf and Y suggest a common source for these metals and identify traffic as the main sources.

The main objective of this research is an attempt to fulfil the lack of knowledge regarding traffic related sources of contamination with PGEs in road dust, mixed sludge and sediment from the gullies as well as in pavement dust in fractions: $<20 \mu \mathrm{m}$ and in bulk sample $(<2000 \mu \mathrm{m})$. In order to assess and observe in the urban environment, sites characterized by heavy traffic and congestions were selected in Krakow, Warsaw as well as in Wroclaw and Opole. The sampling locations were selected to minimize the impact of other PGE sources. They were located as far as possible from industrial plants and, in the case of the motorway, far from residential areas. The investigation were carried out in 2015 and 2016. Metals were extracted from brake lining dust by microwave oven digestion with aqua regia, and samples were digested according to the US EPA method 3050B. PGE contents were determined by ICP-MS (ELAN 6100; Perkin Elmer), according to the US EPA method 6020B. Certified international reference materials BCR 723 was used to ensure that the analytical results met the required criteria. The highest PGEs concentrations were found in road dust samples and mixed sludge and sediment from the gullies. The concentrations of the majority of these elements were significantly elevated when compared with the concentrations found in samples from relatively traffic-unpolluted areas. Moreover, SEM-EDS analysis of road dust have confirmed the presence of catalytic converters components and significantly amount of particles of antopogenic origin.

\section{Acknowledgments}

The research leading to these results has received funding from the Polish-Norwegian Research Programme operated by the National Centre for Research and Development under the Norwegian Financial Mechanism 2009-2014 in the frame of Project Contract No Pol-Nor/208849/106/2015 